Variations sur la lettre, le mètre et la mesure :

Shakespeare

\title{
Shakespeare et la tragédie antique
}

Jean Bollack

\section{(2) OpenEdition}

12 Journals

\section{Édition électronique}

URL : http://journals.openedition.org/shakespeare/969

DOI : 10.4000/shakespeare.969

ISSN : 2271-6424

Éditeur

Société Française Shakespeare

\section{Édition imprimée}

Date de publication : 1 novembre 1996

Pagination : 63-70

Référence électronique

Jean Bollack, « Shakespeare et la tragédie antique », Actes des congrès de la Société française

Shakespeare [En ligne], 14 | 1996, mis en ligne le 01 janvier 2007, consulté le 20 avril 2019. URL : http:// journals.openedition.org/shakespeare/969; DOI : 10.4000/shakespeare.969 


\section{Variations sur la lettre,}

le mètre et la mesure

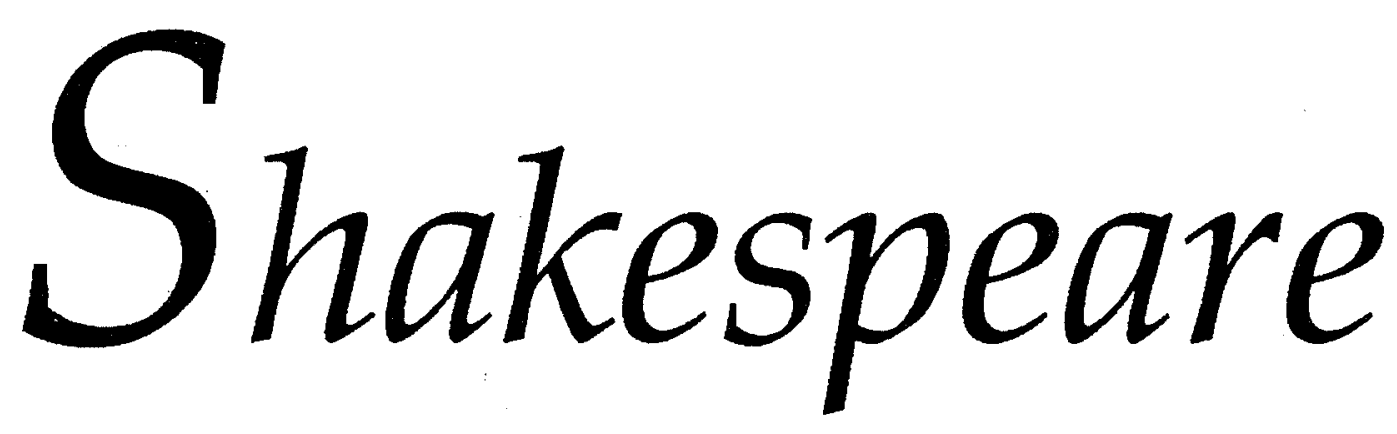

textes présentés par Dominique Goy-Blanquet

Directeur de la publication : Richard Marienstras 


\section{Shakespeare et la tragédie antique}

La tragédie grecque, si j'emploie très provisoirement ce terme générique, se noue sur une action de justice à retardement qui se résout avec la mort, ou pire que la mort : avec le meurtre involontaire. La démesure, si l'on traduit ainsi le mépris de la limite qui fait l'hybris, se casse, éclate dans le néant. C'est le sens donné à l'événement, et donc l'unique nécessité. Une transgression inhérente à une action humaine, et d'abord familiale, se révèle à la fin - "avec le temps", selon la formule; la logique de l'histoire se construit. La durée du temps s'en alimente, elle reste confinée dans cette logique, qui a "sa fin". La découverte éclaire : un "c'était donc...", du côté de la victime, et, pour l'un des dieux, c'était un "avant"; il ne diffère pas de l' "après" qui le confirme. Apollon et Edipe collaborent dans CEdipe roi pour abolir les effets de la transgression du père. L'accord fait que le déroulement de l'action est signifiant, dans un espace déchiffrable et parfaitement clos. Ajax, après son délire, ne peut pas ne pas se suicider. Il n'a pas su maîtriser la blessure d'une injustice.

La forme ne se conçoit pas sans la dualité que lui imprime la présence du chœur comme représentant de l'ordre et de la tradition. La règle est sur scène à côté de l'exception, les défenseurs d'une limite vitale pour la collectivité sont là, à côté d'une aventure et d'un désir d'expansion non réfréné. La cité est ébranlée par la menace, mais son aspiration à la mesure ne l'est pas. Le désordre règne au loin à un autre pôle, près d'une périphérie où s'aventure le héros, dans sa séparation, donnée en spectacle, presque au-delà des confins du monde, lors même qu'il se débat avec les habitants de la cité. L'extériorité est vouée au non-être. Ce vent du néant ne souffle pas moins dans les tragédies de Shakespeare. La différence est dans une sphère, préservée au centre, qui rejette le héros dans l'exception et fait que la tragédie grecque débouche, d'une manière ou d'une autre, sur une interrogation qui concerne le prix payé pour le triomphe de la justice. On retourne ainsi au centre, et à une réflexion sur le sens de cette nécessité qui fait le sens de l'histoire.

Une question transcende ces totalités partielles. Electre s'épuise à jouer l'oiseau noir contre sa mère, à empêcher que le meurtre du père ait servi les plaisirs de Clytemnestre. Mais son combat, si l'on s'en tient à la stricte exécution du droit et du devoir de vengeance, est insensé. Oreste s'est chargé de tout, il le ferait sans elle. La transgression, il semble, touche une révolte que l'on pourait croire légitime. Oreste ne l'aura-t-il pas empêchée de jouer à l'Erinye ? Si c'est le tribut de la vengeance, il se sera retourné contre les exécutants. Electre le reconnait, comme forcée. Antigone, qui agit, est contrainte d'évaluer le prix qu'elle payera pour l'emporter. La tragédie, elle-même, se problématise. 
Ce sont des mythes. Les destins s'agencent dans l'affrontement. Les histoires traditionnelles prennent un sens selon leur structure spécifique, dans le cadre tracé de la mise en évidence scénique d'une transgression au sens propre. Qu'elle connaisse une issue nécessaire, inéluctable, fatale n'est qu'une relecture qui dérive du postulat de la limite. Une structure sémantique se constitue, elle va vers son terme et débouche sur une lamentation et sur une réflexion qui ne se refuse pas à mettre en question le sens qui s'est construit.

Les données de ce cadre, je les tire de Sophocle. C'est une abstraction, nécessairement. Il n'y a de réalité que la pièce singulière, elle-même tọjours complexe. Or cette attente et cette perspective, cette projection, aboutissant à l'issue fatale, ont été abandonnées, sans doute combattues avec acharnement par Euripide, dans un souci sans doute encore) plus artistique, à savoir d'efficacité dramatique, qu'intellectuel ou idéologique, si les deux aspects se laissent distinguer. La nécessité découlant de la logique d'une histoire n'avait pas d'existence pour lui. Le tragique, s'il en existe, ce n'était pas cette démonstration liée à l'échec d'une aventure d'exception. L'expérience de la cruauté ordinaire est bien pire. C'est là que l'hybris se loge, dans les rapports humains qui sont des rapports de puissance, procédant d'une volonté toujours illimitée. Chacun est dans la main d'un autre, qui fait tout pour garder le dessus. Tous les malheurs sont liés à une situation de domination quotidienne que tous les mythes de l'épopée permettent d'illustrer à satiété. Tous les moyens sont bons. S'ils réussissent: on apprécie les connaissances. Le dépassement des limites, on le savait, est la conduite naturelle, il faut dire ordinaire. Le degré est aussi arbitraire que l'est le pouvoir qui le fixe. La tragédie se joue entre forts et faibles. Dans l'histoire du métier, une forme s'était constituée au regard de laquelle l'autre, l'ancienne, devait apparaître comme une entreprise arbitraire.

Que pouvait faire Médée, dans la situation indépassablement vraie où elle s'est trouvée, sacrifiée aux conventions et aux intérêts les plus sordides et les plus normaux ? Elle n'avait d'autre issue que de se venger en se détruisant - un peu comme luttait Antigone en mettant sa propre mort dans la balance. Médée fait avec ce qu'elle a, pour faire mal. C'est ainsi qu'a son tour elle est plus forte que l'autre. C'est, si l'on veut, une riposte, celle de la femme délaissée devant le sarcasme qu'est pour elle la conduite rangée de son amant, de la chair contre la raison, non moins cruelle. Le désespoir et le refus de se soumettre font de la vengeance la vérité sociale et physique la plus crue. Personne n'a rien décrété là, ni homme ni dieu, sinon la violence de la lutte qui fait la loi chez les hommes et engendre les actions comme des réactions. Médée dans l'entourage de Corinthe, où l'a amenée Jason, n'est plus un parti pour lui, elle n'y est rien, comme Andromaque n'est rien dans le palais de Phthie, avant l'arrivée de Pélée, capable de la protéger, et la protégeant parce qu'il lui plaît de le faire. Ce n'est pas beau.

La première manière ne survit pas dans Shakespeare. Aucun ordre n'est rétabli par aucune expiation. Dans Titus Andronicus, à la fin, tous les acteurs du drame se tuent les uns les autres en chaîne, les bons et les mauvais, comme à la fin de Hamlet. La justice est aussi factice que l'arrivée de Lucius à la tête d'une armée de Goths. La seconde manière introduite par Euripide a triomphé. Le châtiment en règle est l'arme d'un pouvoir comme le crime l'est d'un autre : ils sont presque 
échangeables. Le triomphe est un état de fait, au-delà des siècles. Le théâtre, c'était devenu cela. Shakespeare ne pouvait pas en avoir conscience. Du moins n'avait-il pas su attribuer à des formes constituées la différence qu'il fait, lui, en choisissant une matière qui lui a permis d'aller beaucoup plus loin. Euripide refaçonnait, il réinventait les sujets connus, le mythe traditionnel, il restait donc confiné dans le cadre d'une intrigue, stylisée, plus littéraire que romanesque, qui ne s'ouvrait pas sur la grande histoire. La vérité des luttes et des catastrophes nous déplace jusqu'au lieu du mal; il reste à peine localisable, s'accroissant comme il veut. Aucune histoire autre que celle qui nous emporte ne peut lui donner un visage. Il portera tous les masques.

Shakespeare, par ce biais et ce dépassement, est bien le continuateur de la tragédie grecque. Il s'est inscrit dans son évolution ; il a traversé une histoire du genre qui la continue, et dont Euripide, sans conteste, a été l'instigateur. Toutes les médiations ont été marquées par lui. Le thêâtre moderne, c'est lui qui l'a créé au moyen des transformations qu'il a fait subir au genre. Les choses se font du dedans. L'ouverture est tributaire de la négation du sens, qui instaure le privilège de l'invention sur tout le reste. Quoi de plus beau que la main souveraine de l'art qui se fait voir ? Il faut dire que la manière s'est imposée, parce qu'elle était mieux adaptée à un public qui s'était transformé, et qu'elle était plus forte. Tout est euripidéen à la fin de l'Antiquité, tout le répertoire au sens le plus technique. "Le poète", c'était lui, le maître de l'affect, capable de détrôner Homère, du moins de rivaliser avec lui, parce que leurs interventions, au sein d'une tradition forte, avaient été comparables.

A l'époque de Shakespeare, on dit Sénèque; c'est souvent Euripide que sans le savoir on désigne. Aujourd'hui, la tragédie, c'est plutôt Eschyle et Sophocle. Nous sommes en cela les héritiers encore du XIX ${ }^{\mathrm{e}}$ siècle. On ne s'est pas intéressé à la mise à nu ni à l'analyse, ni aux jeux factices. Euripide n'a pas été joué au théâtre (quasiment pas, jusqu'à une date récente). Il n'en avait pas été ainsi pour Racine, sensible à ses qualités dramatiques et à la modernité de son art — qu'il ne nommait pas ainsi. Goethe, de son côté, sentait bien que sans lui il n'y avait pas de théâtre possible ; aussi le défendait-il avec force contre ses nombreux détracteurs, bien que par ailleurs il reconnût lui-même la différence profonde. Sophocle, c'était "la nature", à savoir l'intuition des lois profondes et permanentes, Euripide, ce n'était que "l'art", qu'il fallait donc revaloriser - helléniser.

Chez Shakespeare, la tragédie grecque semble une absente, et c'est là le fait signifiant, qu'il faut interpréter. Il n'a écrit ni une Electre ni une Médée. On pourrait se dire que la différence ne serait pas moindre, s'il en avait écrit une au terme de deux mille ans. Ce n'est pas sûr. On ne voit pas la manière dont il aurait repris ces sujets, ni la forme de lecture, ni donc la transformation et le remaniement.

Pour déterminé que soit ce manque par sa formation et l'horizon intellectuel du temps, il n'est pas moins signifiant. J'essaye de donner un sens à une absence, un révélateur peut-être, si l'on tente de reconstruire ce que la tragédie grecque avait pu représenter dans l'imagination de Shakespeare, non seulement ce qu'il a pu en connaître, mais ce qu'il a pu en penser. On en arrive à une idée que l'on tire à la fois de l'œuvre du poète, et de la chose, et qui doit faire comprendre pourquoi il a évité 
la matière. C'est une hypothèse, mais si elle se confirme, elle permet de dépasser le constat d'un manque fortuit.

Ce n'est donc pas l'absence d'une forme de la tragédie, que les théoriciens ont construite d'après le modèle grec, lorsqu'elle n'incluait pas expressément Shakespeare, que j'envisage, mais l'impossibilité d'un sujet adéquat. Timon, c'est autre chose, la fable appartient à un autre monde, et Troilus et Cressida s'éloigne plus encore de la matière du théâtre grec, bien que ce soit l'unique pièce qui fasse implicitement référence à l'une des grandes œuvres de la littérature grecque. Le mythe n'était plus, ou n'était pas pour lui un thème dramatique possible. On connaissait les Métamorphoses, ces Mille et une nuits de la mythologie ; on pouvait (on avait pu) parfois pousser plus loin et pénétrer par d'autres lectures jusqu'aux secrets et aux complexités des drames. Mais ce n'était plus sérieux. On ne faisait que donner corps à un horizon constellé de merveilles qui distinguait les lettrés. La mythologie était omniprésente, mais elle appartenait à l'ordre du langage, à un système de figures qui offrait à une rhétorique précieuse d'inépuisables ressources. La Grèce avait cette image, et n'était-il pas vrai que sa littérature se confondait largement avec les histoires trop enjouées, lors même qu'elles étaient tristes, de cet univers mythologique? Elle était le mythe même, et par là, discréditée pour la scène - et pas seulement la scène tragique. Le mythe florissait dans les sphères aériennes de la poésie; il ornait, et croisait, et parfois creusait le drame; mais je n'hésiterais pas à y inclure le type d'histoires qui avait fourni sa logique et son sens à la tragédie. Ce qui était récusé, c'était simplement ce que l'on considérait comme un enchaînement prédéterminé trop rigide. Il suffit de dire : une construction signifiante, pour comprendre qu'elle ait dû être récusée devant l'appel d'un mouvement de l'histoire que les tragédies romaines font sentir. On recommencera ailleurs. La libération qu'avait opérée Euripide ne pouvait être perçue et distinguée du reste. C'était une opposition interne, inhérente à un genre dépassé. Son art agissait ailleurs, dans les comédies de Térence, dans les tragédies de Sénèque, dans Shakespeare.

L'exil d'Edipe à Corinthe dans Edipe roi, la phase la plus vraie et la plus fausse de son existence, suivie d'une autre plus trompeuse encore, mais vraie, prend son sens dans le cadre définitionnel d'une problématique qui demande à être déchiffrée. Shakespeare nous jette dans un univers différent avec l'exil du fils de Titus ou celui que choisit Coriolan. Ils quittent la ville pour leur bien ou leur perte, ils s'en vont et reviennent, ou ne reviennent pas. C'est sans doute chaque fois une action libre, et non un leurre, couvant une signification obscure, ou simplement un acte. L'acteur cherche à se soustraire à une détermination, ou non, il se précipite vers une destinée prévisible, ou non. C'est entre les deux. On mesure l'angle de l'ouverture qui est grand, on pourrait encore, entre les deux, dire : immense et nul.

Dans le losange que construisent les quatre tragédies romaines, il y a une origine et une fin, avec au centre le double conflit de l'apogée (cela n'a pas été conçu comme cela, mais c'est un aboutissement, une téléologie de l'œuvre), l'événement se produit pour que l'histoire puisse s'affirmer. Faut-il retenir l'issue, que l'on retient? Et pas plutôt l'antagonisme, les bannissements plus vrais, les affrontements, la lutte 
pour le pouvoir, l'invasion des barbares? Le sens est dans l'issue, qui n'est pas le sens, mais la lutte. La situation d'Euripide, la seule réalité, prime, mais elle n'est plus séparée de l'histoire. C'est donc elle, avec son absurdité, qui fait sens. Walter Benjamin, dans son livre sur la tragédie baroque en Allemagne (le Trauerspiel), a beaucoup insisté sur l'importance des chroniques. Il y a là quelque chose de plus élémentaire et violent, de plus radical encore dans cette substitution offerte à la matière grecque. Le combat est attribué au rapport de forces. La base a été jetée là. Rome, c'est l'entrée dans une histoire, l'Empire christianisé, qui se perpétue dans la nôtre, le Moyen Age français et anglais, un monde différent, comme paradigmatique, qui a disparu, mais n'a rien de mythique. Ce n'est pas la clé de voûte, comme on l'a souvent affirmé, d'une philosophie de l'histoire, bien plus une référence politique et culturelle, culturelle parce que politique, de premier ordre.

La culture de l'époque joue son rôle, qui est primordial. Mais le génie ou plus simplement une capacité extraordinaire, font qu'une fraîcheur nouvelle perce, ou les reprises les plus attendues. Aussi la détermination est-elle ancienne et brisée. Il y a lieu de distinguer de l'expérience qu'une situation culturelle permet de faire, les conditions de son dépassement. Si l'on prend la base de l'histoire romaine, on dira que si l'imprégnation des esprits à la fin du XVI $\mathrm{X}^{\mathrm{e}}$ siècle est latine, la signification qui est donnée à ce choix reste spécifique; et s'il est vrai que les sujets grecs sont extrêmement rares dans le théâtre britannique de l'époque - beaucoup plus rares qu'en France, l'option n'est pas moins instructive.

Les conditions historiques, la formation scolaire ou la diffusion des livres délimitent l'étendue de l'information. Qu'est-ce qui était connu ou accessible ? Charles et Michelle Martindale ${ }^{1}$ notent que $\mathrm{Velz}^{2}$, dans son état de la question de 1978 consignait déjà 2487 items sur la question - et tout va en croissant. Avant de reprendre le problème, qui dépasse celui de l'humanisme, il faut définir ou redéfinir la notion de "source", et s'entendre sur ce que l'on compare, et sur ce que l'on cherche à démontrer. La question est complexe. Comment l'horizon intellectuel et littéraire se constituait-il ? La connaissance d'un texte précis semble s'imposer. Les médiations sont multiples, elles oscillent entre l'évident et le possible.

Il convient de mettre la maitrise du langage au premier plan. La langue de Shakespeare tend à s'autonomiser sans cesse au cours de l'action dramatique, elle se regarde faire. On prend alors la mesure de l'ampleur des références - des lectures dues à la curiosité de l'auteur qui, en écrivant, reprend des textes pour les réécrire. La maîtrise s'appuie sur une rhétorique souveraine, nourrie à l'antique, et donc, par cet apprentissage, sur une culture livresque déjà rhétorisée et poétisée. Les résidus et les extraits de tous les textes sont virtuellement présents dans ce tout-ensemble, s'offrant à l'argumentation et à la persuasion mais tout aussi bien au jeu des créations gratuites et virtuoses. Le grec, on peut en trouver dans les auteurs latins. qui jouissent d'un statut particulier parce qu'ils sont proches, à moitié étrangers seulement, et que le bien-dire se constitue à travers eux. C'est avec du latin que l'on fait l'anglais de North quand il traduit Plutarque du français d'Amyot. Le grec n'attend pas d'être lu parce qu'il n'est pas intégrable au même titre, tant s'en faut, que le latin qui fait comme partie de l'anglais et du français et attend d'être dit autrement. La formation initiale est connue par les admirables travaux de Baldwin 
sur le cursus des grammar schools, et leurs programmes. L'apprentissage d'une langue créait le plaisir d'y exceller; mais comme indépendamment - que l'on songe aux scènes de salon de musique verbale - on entend s'élever une autre langue encore, précieuse comme tout ce qui irradie, se détachant des strates plus rudes et vulgaires. L'art de cet art, à savoir l'artifice, repose sur d'autres lectures identifiées ou identifiables. Il faut en majorer plutôt qu'en minorer le nombre.

Il en va du théâtre comme de la langue. On imagine un lexique, un répertoire de toutes les situations possibles. L'instrument de travail, quelle qu'en fût la forme, devait faire partie du métier (je ne sais pas ce que l'on connaît dans le genre). On travaillait sur des textes non dramatiques, le plus souvent narratifs. Ils fournissaient une trame et parfois, comme les biographies de Plutarque, les éléments de l'adaptation, des fragments de discours. Mais ce qui structure et fait progresser l'action, ce serait plutôt cette typologie des scènes telle qu'on peut la concevoir, une grammaire des situations, longues ou concentrées, ouverte ou fantastique. Comme on apprend à modeler des figurines et à les mettre en place. On a observé qu'à quelques exceptions près la plupart des scènes (ou des situations) se retrouvent variées d'une des pièces à l'autre. Cela correspond à l'impression de chaque lecteur, s'il lit les pièces à la file, mais une étude systématique aboutirait à dresser un inventaire où serait répertoriée une large partie de l'histoire du théâtre, théâtre antique compris, lors même que les œuvres n'étaient pas connues ou accessibles directement.

Les chapitres consacrés à Euripide dans le livre d'Emrys Jones, The Origins of Shakespeare (1977), illustrent bien cette distinction entre les éléments compositionnels et le travail sur les citations textuelles, du côté de l'invention verbale. Jones, du côté du théâtre, a montré que le début d'Iphigénie à Aulis où, au cours de deux scènes successives, Ménélas invective son frère avant de s'apitoyer sur lui, fournit le modèle d'un mouvement similaire de retournement que Dryden a introduit dans son adaptation de Titus, mais surtout que Shakespeare avait utilisé pour la grande scène de discussion entre Brutus et Cassius dans Jules César. C'est la singularité du canevas, dans l'œuvre, qui est remarquable. Erasme avait traduit cette Iphigénie, mais aussi Hécube, dont la structure, avec le passage de la femme victime et pitoyable au statut de femme vengeresse et enragée a fourni la figure de l'évolution dramatique dans Titus. On pourrait être conduit à supposer le contenu de l'information par une étude des structures. La vraisemblance qu'il ait connu ces pièces consolide l'hypothèse. Elles étaient, avec les Phéniciennes, les plus appréciées par les humanistes (chaque génération a refait son Euripide, et ce n'était jamais le bon — mais celui qu'il fallait).

L'histoire de l'interprétation est centrale, les moyens de l'étudier ne manquent pas. L'histoire, quand elle a été décrite, conduit rarement jusqu'à reconnaître les écrans que les attentes ont dressés entre l'œuvre et le lecteur. Un sujet comme celui du "classical" background est fortement lié à celui, plus général, du classicisme. On pouvait vouloir retrouver dans Shakespeare une forme idéale de la tragédie si elle était héritée et si elle remontait à l'Antiquité, je dis bien selon le modèle que l'on s'était construit, et que le plus souvent on avait emprunté aux théoriciens hellénisants du XIX ${ }^{\mathrm{e}}$ siècle. Mais on disposerait d'autant d'évidences pour soutenir le 
point de vue contraire et voir en lui le champion de l'émancipation artistique, le représentant d'une modernité en rupture d'idéal et de règles. Comme par nécessité, la synthèse devait prévaloir. Le cas de Shakespeare, lorsque son importance a été reconnue, même en France, était si éclatant que son classicisme pouvait être sanctionné au moyen de la différence. L'incompatibilité s'annulait devant la force. On retrouvait ce qu'on connaissait, le plaisir d'un couple qui s'était constitué par un meurtre, Clytemnestre et Egisthe, l'Erinye rôdant dans le palais comme Hamlet sur le modèle d'Electre, bien plus que d'Oreste - si l'on s'en tient à la pièce de Sophocle - et pourtant Shakespeare n'avait pas suivi ni même renouvelé ou ravivé les modèles. C'est donc qu'il existait une virtualité de forme plus générale, plus essentielle et comme transhistorique qui s'était épanouie à des moments différents. A ce compte, Shakespeeare n'était pas moins grec que les Grecs, ou il l'était même davantage, appelé à fournir un modèle plus juste si la reprise d'une incarnation antérieure ou première traduisait une inspiration plus libre et plus forte. On peut toujours dire, en privilégiant la constance, que l'acte créateur, en se reproduisant, se saisit des mêmes motifs, qu'ils sont dans la nature, à savoir dans l'inspiration, et non dans les livres et le savoir-faire. En croyant à l'inspiration naturelle, on diminue l'importance des connaissances, au profit des découvertes spontanées. C'est une lecture, la façon de lire et de ne pas lire, qui s'y révèle. L'appréciation des points de vues permet de comprendre l'étendue des connaissances; elle est tributaire d'un jugement sur la nature du texte, sur la liberté du langage. Tout est transformation, et suppose un état préexistant, et peut-être pour le lecteur-spectateur une connaissance de cette antériorité pour appréhender la différence.

Jean BOLLACK

Université de Lille-III

1 Shakespeare 's Use of Antiquity: An Introductory Essay, Londres et New York, 1990.

2 John W. Velz, The Ancient World in Shakespeare : Authenticity or anachronism? A retrospect, 1978. 